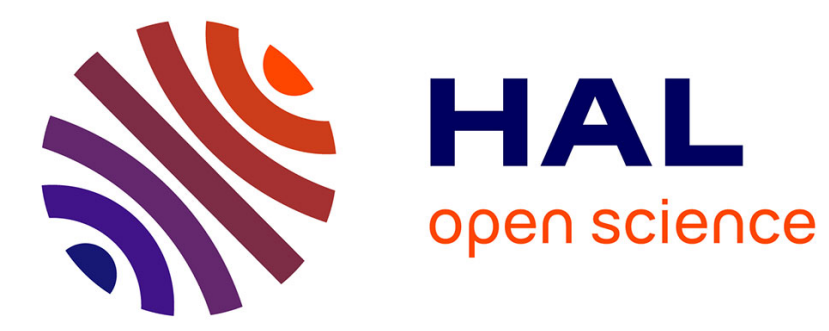

\title{
microRNA-directed cleavage and translational repression of the copper chaperone for superoxide dismutase mRNA in Arabidopsis
}

Linda Beauclair, Agnes Yu, Nicolas Bouche

\section{To cite this version:}

Linda Beauclair, Agnes Yu, Nicolas Bouche. microRNA-directed cleavage and translational repression of the copper chaperone for superoxide dismutase mRNA in Arabidopsis. Plant Journal, 2010, 62 (3), pp.454-462. 10.1111/j.1365-313X.2010.04162.x . hal-01203867

\section{HAL Id: hal-01203867 \\ https://hal.science/hal-01203867}

Submitted on 31 May 2020

HAL is a multi-disciplinary open access archive for the deposit and dissemination of scientific research documents, whether they are published or not. The documents may come from teaching and research institutions in France or abroad, or from public or private research centers.
L'archive ouverte pluridisciplinaire $\mathbf{H A L}$, est destinée au dépôt et à la diffusion de documents scientifiques de niveau recherche, publiés ou non, émanant des établissements d'enseignement et de recherche français ou étrangers, des laboratoires publics ou privés. 


\title{
microRNA-directed cleavage and translational repression of the copper chaperone for superoxide dismutase mRNA in Arabidopsis
}

\author{
Linda Beauclair ${ }^{1}$, Agnès $\mathrm{Yu}^{2, \dagger}$ and Nicolas Bouché ${ }^{1, *}$ \\ ${ }^{1}$ Institut Jean Pierre Bourgin, UMR1318 INRA-AgroParisTech, INRA Centre de Versailles-Grignon, route de St Cyr, \\ F-78026 Versailles, France, and \\ ${ }^{2}$ URGV, UMR INRA 1165 - CNRS 8114 - UEVE, 2 rue Gaston Crémieux, F-91057 Evry, France
}

Received 4 January 2010; accepted 20 January 2010; published online 12 March 2010.

*For correspondence (fax +33 1308333 19; e-mail bouche@versailles.inra.fr).

${ }^{\dagger}$ Present address: Institut de Biologie Moléculaire des Plantes du CNRS, Unité Propre de Recherche 2357, 12 rue du Général Zimmer, F-67084 Strasbourg, France.

\begin{abstract}
SUMMARY
microRNA398 (miR398) is a conserved miRNA of plants that targets two of the three copper/zinc superoxide dismutases (SOD) of Arabidopsis (CSD1 and CSD2) by triggering cleavage or inhibiting translation of their mRNAs. We analysed the transcriptomes of mutants impaired in miR398 production, and found that the mRNAs encoding the copper chaperone for superoxide dismutase (CCS1), which delivers copper to CSD1 and CSD2 apoproteins in different cellular compartments, are undiscovered targets of miR398. We identified the cleavage site in CCS1 mRNAs by 5'-RACE PCR. We further show that both CCS1 protein and mRNA levels are tightly linked to the quantities of miR398, which are themselves dependent on the copper content in the medium. We generated transgenic plants carrying a CCS1 mRNA version resistant to cleavage by miR398, and demonstrated that both CCS1 mRNAs and proteins accumulate in these plants when miR398 is abundant and copper limiting. Moreover, we show that one of the ten ARGONAUTE proteins of Arabidopsis (AG010) is involved in miR398-directed translational inhibition of CCS1 mRNAs, as CCS1 protein, but not CCS1 mRNAs accumulates in ago 10 ( $z / l)$ mutants. Thus, miR398 mediates the cleavage and translational inhibition of mRNAs encoding CCS1, the chaperone protein that is essential for generating the mature copper/zinc SODs of Arabidopsis. Our results also imply that new targets that have not been identified by computing analyses have yet to be discovered, even for an extensively studied miRNA such as miR398.
\end{abstract}

Keywords: copper chaperone for superoxide dismutase (CCS1), copper/zinc superoxide dismutase, miR398, ARGONAUTE, Arabidopsis.

\section{INTRODUCTION}

microRNAs (miRNAs) are a class of small RNA regulators of 21-24 nucleotides that play key roles in developmental processes of both plants and animals by directing cleavage or translational inhibition of target mRNAs (Mallory and Bouché, 2008; Voinnet, 2009). miRNAs are processed from imperfectly paired hairpin precursors derived from transcription of MIR genes by RNA polymerase II. miRNAs direct a variety of developmental events in plants, and there is increasing evidence that points to potential roles in diverse physiological processes. For instance, miR395 and miR399 are involved in sulfate and phosphate deficiencies, respectively (Chiou, 2007), and miR398 appears to be involved in responses to environmental stresses (Sunkar et al., 2006; Jia et al., 2009; Trindade et al., 2010).
miR398 is a conserved miRNA that was identified in Arabidopsis by both computational analyses (Bonnet et al., 2004; Jones-Rhoades and Bartel, 2004) and sequencing of small RNA libraries from seedlings exposed to abiotic stresses (Sunkar and Zhu, 2004). Based on sequence homologies, miR398 was predicted to target mRNAs of both cytosolic CSD1 (copper/zinc superoxide dismutase 1) and chloroplastic CSD2, as well as a subunit of the mitochondrial cytochrome $c$ oxidase, COX5b-1 (Bonnet et al., 2004; JonesRhoades and Bartel, 2004; Sunkar and Zhu, 2004). Cleavage of the target mRNAs was experimentally verified (JonesRhoades and Bartel, 2004) using a modified version of 5'-RACE PCR (Llave et al., 2002). When plants are exposed to abiotic stresses such as high levels of $\mathrm{Cu}^{2+}, \mathrm{Fe}^{3+}$, ozone or Journal compilation @ 2010 Blackwell Publishing Ltd 
salt, or to biotic stresses such as a bacterial infection by Pseudomonas, the levels of miR398 decrease (Sunkar et al., 2006; Jagadeeswaran et al., 2009). For instance, at least two of the three MIR genes encoding miR398 (i.e. MIR398b and MIR398c) are transcribed only when copper is limiting (Sunkar et al., 2006; Dugas and Bartel, 2008), in contrast to the CSD2 gene, which is transcribed at the same rate under low- and high-copper conditions (Yamasaki et al., 2007). However, CSD2 mRNA is down regulated when copper is low, implying a post-transcriptional control that depends on copper availability. miR398 plays an essential role in this process, as demonstrated firstly by generating transgenic plants expressing modified versions of CSD1 or CSD2 mRNAs rendered resistant to cleavage by miR398, thus artificially increasing target mRNA levels in these plants (Dugas and Bartel, 2008), and secondly, by over-expressing MIR398 precursors leading to the opposite phenomenon (Yamasaki et al., 2007; Dugas and Bartel, 2008). In addition to transcript cleavage, miR398 also acts through translational repression of the target mRNAs when copper is limiting (Brodersen et al., 2008). The exact molecular mechanism underlying this process remains to be elucidated, but probably involves two of the ten ARGONAUTE (AGO) proteins of Arabidopsis, AG01 and AGO10 (Brodersen et al., 2008). Indeed, ago10 mutations restore leaf development and siRNA and miRNA pathways in ago 1 hypomorphic mutants and lead to increased AGO1 protein levels, thus implicating AGO10 as a negative regulator of AGO1 (Mallory et al., 2009). Moreover, analyses of chimeric gene constructs indicate that the PAZ domain, which is thought to mediate small RNA binding, is exchangeable between both proteins, whereas the MID-PIWI and N-terminal domains appear to contribute to their specific functions (Mallory et al., 2009). Very recently, Lanet et al. (2009) provided biochemical evidence for translational repression by showing that active miRNAs associate with polysomes via their target mRNAs.

In Arabidopsis, the copper cofactor is delivered to $\mathrm{Cu} / \mathrm{Zn}$ superoxide dismutases (SODs) by a copper chaperone for superoxide dismutase (CCS1) (Abdel-Ghany et al., 2005; Chu et al., 2005). The Arabidopsis genome contains a unique CCS1 gene encoding both a cytosolic and a chloroplastic form of CCS1, which deliver copper to SODs localized in different cellular compartments. Here, we show that miR398 directs the post-transcriptional regulation of CCS1 mRNAs by cleavage and AG010-mediated translational repression.

\section{RESULTS}

\section{Isolation and molecular characterization of mutants with altered levels of miR398}

In Arabidopsis, the miR398 family is represented by two members that differ by one nucleotide at the $3^{\prime}$ end and are encoded by three genes, namely MIR398a, MIR398b and MIR398c (Bonnet et al., 2004; Jones-Rhoades and Bartel,

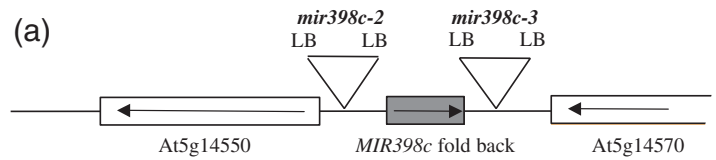

(b)

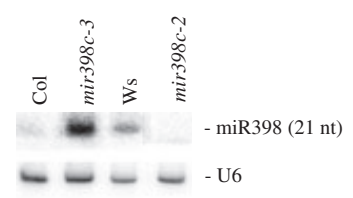

Figure 1. Levels of miR398 in mir398c mutants.

(a) Schematic presentation of MIR398c and positions of the T-DNA inserts in mir398c-2 (FLAG_072C02) and mir398c-3 (SALK_017704) mutants, represented by triangles. LB, sequenced left borders of the T-DNA inserts.

(b) RNA gel blot analysis of $10 \mu \mathrm{g}$ total RNA prepared from rosette leaves of Col and Ws wild-type plants and mir398c-2 and mir398c-3 mutants. U6 RNA was used as a loading control.

2004; Sunkar and Zhu, 2004). Two mutations in the Arabidopsis MIR398 loci have been described previously (Dugas and Bartel, 2008): mir398a-1 (SALK_043101) and mir398c-1 (SALK_039698). By comparing the MIR398 sequences with T-DNA flanking genomic sequences deposited in the databases, we isolated additional T-DNA knockouts corresponding to putative mir398 mutants. Their genomic DNAs were characterized by PCR, and the junctions between the T-DNAs and the MIR398 genes were sequenced. Plants homozygous for the mutations were isolated, and the levels of miR398 were determined by Northern blot analysis. Plants were grown for 30 days on soil, and total RNAs from rosette leaves of five plants per homozygous line were extracted in bulk, blotted on a membrane and hybridized with an miR398-specific probe. We found two mutant alleles with T-DNAs inserted in MIR398c (Figure 1a) that contained lower and higher levels of miR398 compared to their respective wild-type (Figure 1b). Similar results were obtained with total RNAs extracted from flowers of the same plants (data not shown), and this experiment was repeated three times under the same conditions. In mir398c-2 (FLAG_072C02), the global production of miR398 is impaired by an unknown mechanism, as MIR398b and/or c precursors appear to be normally produced in these plants (Figure S1). In contrast, both miR398 (Figure 1b) and MIR398b and/or c precursors (Figure S1) accumulate in mir398c-3 (SALK_017704), probably because of enhancer sequences carried by the T-DNAs, such as CaMV 35S enhancers.

\section{Transcriptome analyses of $m i R 398 \mathrm{c}$ mutants reveal a new target of miR398}

mir398c mutants altered in the production of miR398 were then used to identify target mRNAs of miR398 by analysing their transcriptomes. We hypothesized that target transcripts would be up-regulated in mir398c-2 and downregulated in mir398c-3. Ten to fifteen mir398c-2, mir398c-3 
Table 1 List of genes up-regulated in mir398c-2 and down-regulated in mir398c-3

\begin{tabular}{llll}
\hline $\begin{array}{l}\text { AGl gene } \\
\text { accession }\end{array}$ & Function & $\begin{array}{l}\log _{2} \\
(\text { mir398c-2/Ws })\end{array}$ & $\begin{array}{l}\log _{2} \\
(\text { mir398c-3/Col) }\end{array}$ \\
\hline AT1G08830 & CSD1 & +0.79 & -1.7 \\
AT1G12520 & CCS1 & +0.96 & -1.41 \\
AT1G78370 & Glutathione & +0.82 & -0.71 \\
& transferase & & -1.23 \\
AT2G28190 & CSD2 & +1.08 & -1.36 \\
AT5G08640 & FLS1 (flavonol & +0.75 & -1.45 \\
& synthase 1) & & \\
AT5G13930 & Chalcone & +1.14 & \\
& synthase & &
\end{tabular}

Transcriptome analyses were performed using the CATMA microarray on RNAs extracted from rosette leaves of mir398c-2, mir398c-3 and wild-type plants. The results with a Bonferroni $P$ value $<0.05$ confirmed in two independent biological repeats are presented as $\log _{2}$ ratios. AGI, Arabidopsis Genome Initiative.

and wild-type plants were grown in culture chambers under standard growth conditions for 17 days. Rosette leaves from each plant were pooled, and total RNA was extracted in bulk for comparison by microarray analysis. We found only six mRNAs that were up-regulated in mir398c-2 compared to Ws and also down-regulated in mir398c-3 compared to Col (Table 1). Two of those were targets of miR398 characterized previously, namely the CSD1 and CSD2 mRNAs (Sunkar et al., 2006). We compared the coding sequences of the four remaining putative targets to the sequence of miR398a using the RNAhybrid program, which identifies the energetically most favorable hybridization sites of a small RNA in a large RNA (Rehmsmeier et al., 2004; Kruger and Rehmsmeier, 2006). Only one putative mRNA target, corresponding to CCS1 (At1g12520), formed duplexes with miR398 that were predicted to be as stable as the ones formed by CSD1 or CSD2 mRNAs with miR398 (Table 2 and Figure S2). We therefore concluded that CCS1 mRNAs are probably targeted by miR398. This was further confirmed using a modified 5'-RACE PCR technique that enables precise determination of the cleavage sites of miRNAs on their mRNA targets (Llave et al., 2002). As already observed for many other targets of miRNAs in Arabidopsis (Mallory and
Bouché, 2008), we found that CCS1 mRNAs were cleaved within the coding sequence at the predicted miR398 target site, between nucleotides 10 and 11 of the miRNA (Figure 2a). As CCS1 is transcribed in various spliced forms (Chu et al., 2005), and as all of them contain the last exon of CCS1, it is likely that miR398 targets all mRNA forms of CCS1 in Arabidopsis. We aligned the CCS1 genomic sequences retrieved from databases for various plant species, and found that the miR398-complementary site was conserved in all sequences (Figure $2 b$ ).

\section{CCS1 mRNAs and protein abundance are determined by the level of miR398}

When copper is limiting, MIR398b and MIR398c genes are expressed, as shown by MIR398 promoter::GUS transcriptional fusion analyses (Figure 3a) (Sunkar et al., 2006; Dugas and Bartel, 2008). Under these conditions, miR398 levels remain high, and, as a consequence, both CSD1 and CSD2 transcripts are cleaved, resulting in low amounts of proteins (Dugas and Bartel, 2008). To determine whether the abundance of CCS1 mRNAs is post-transcriptionally regulated by copper availability, we grew mir398c-2 and corresponding wild-type sibling plants on plates supplemented with various amounts of copper $\left(0,0.1,0.2\right.$ and $0.3 \mu \mathrm{m}$ of $\mathrm{CuSO}_{4}$ added to the medium). After 15 days of growth, total RNAs and proteins were extracted in bulk from 10 to 15 plants. Levels of miR398 in the wild-type were determined by Northern blot analyses, and the results showed that the miRNA quantity decreases when copper is added to the medium and becomes undetectable at $0.3 \mu \mathrm{m}$ of $\mathrm{CuSO}_{4}$ (Figure 3b). Compared to the wild-type siblings, mir398c plants contain lower amounts of miR398 at 0, 0.1 and $0.2 \mu \mathrm{m}$ of $\mathrm{CuSO}_{4}$ (Figure 3b). These results confirmed that mir398c2 plants are impaired in miR398 production, and that levels of miR398 depend on copper availability in the medium. CCS1 mRNA levels, determined by quantitiative RT-PCR and compared to actin mRNA levels, were tightly linked to the quantities of miR398 in both mutant and wild-type plants (Figure 3c). This was also the case when sucrose was added to the medium (Figure S3). Similarly, CCS1 protein quantities detected by Western blotting and a CCS1-specific

Table 2 Characteristics of the duplexes formed by miR398 and target mRNAs validated by $5^{\prime}$-RACE PCR

\begin{tabular}{|c|c|c|c|c|c|}
\hline \multirow[b]{2}{*}{ mRNA target } & \multirow[b]{2}{*}{ Duplex MFE (kcal/mol) ${ }^{a}$} & \multicolumn{2}{|c|}{ Number of mismatches } & \multirow{2}{*}{$\begin{array}{l}\text { Number of GU wobbles } \\
\text { In the seed region }{ }^{\mathrm{b}}\end{array}$} & \multirow[b]{2}{*}{ Outside the seed region ${ }^{b}$} \\
\hline & & miR398a & $\mathrm{miR} 398 \mathrm{~b} / \mathrm{c}$ & & \\
\hline CSD1 & -34.7 & 3 & 4 & 0 & 0 \\
\hline CSD2 & -34.7 & 3 & 2 & 1 & 2 \\
\hline CCS1 & -33.3 & 4 & 5 & 1 & 0 \\
\hline cox5b-1 & -36 & 2 & 3 & 0 & 0 \\
\hline
\end{tabular}

a Values for MFE (maximum free energy) were obtained using the RNAhybrid program (Rehmsmeier et al., 2004; Kruger and Rehmsmeier, 2006) with miR398a. The MFE of a perfectly match hybrid of miR398a with itself is $45.7 \mathrm{kcal} / \mathrm{mol}$ and the MFE of a perfectly matched hybrid of miR398b/c with itself is $46.9 \mathrm{kcal} / \mathrm{mol}$.

${ }^{\mathrm{b}}$ The seed region corresponds to nucleotides 2-6 starting from the $5^{\prime}$ end of the miRNA. 
Figure 2. miR398 targets CCS1 mRNA.

(a) Schematic presentation of the CCS1 gene (drawn to scale). The location of the miR398 target site in CCS1 is shown. Exons are represented by white boxes and UTRs by grey boxes. The sequence (Col-0) of the miR398 recognition site in the CCS1 mRNAs is aligned with sequences of miR398a and miR398b/c. Cleavage was experimentally validated using a modified version of $5^{\prime}$-RACE PCR (Llave et al., 2002). The arrow indicates the $5^{\prime}$ terminus cleavage product for the number of clones indicated.

(b) Sequences of the CCS1 miR398-complementary site in various plant species. The sequence accession numbers are given in parentheses. Nucleotides matching either miR398a or $\mathrm{miR398b/c}$ are shown in bold. (a)

CCS1 At1g12520.1

(b)

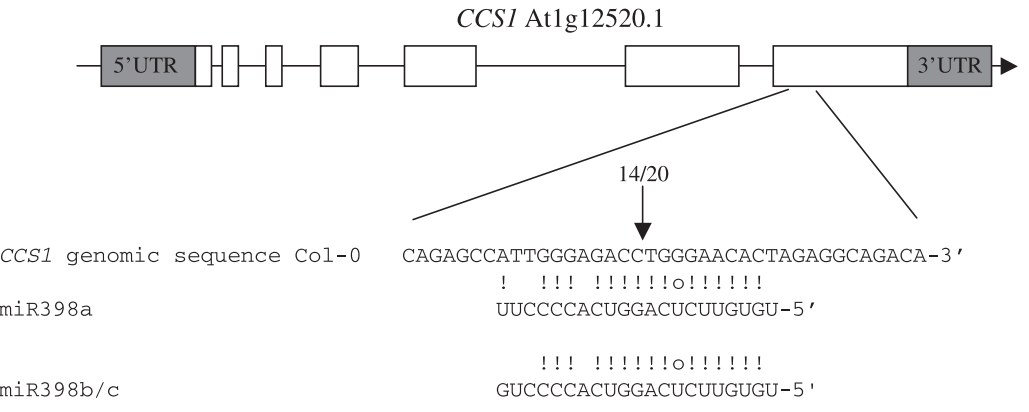

Col-0

Glycine max (AF329816) AAAACAGTAAAGAGCCACTGGGCGACCTGGGACACTAGAAGCTAACG-3

L.esculent. (AF117707)

O. sativa (NM_001060148)

S. 1ycop. (AP009276)

. vinifera (AM435789)

tubero. (AY196210)

trunc. (AC137510)

habro. (AY941689)

Chilense (AY941679)

peruvian. (AY941673)

pimpine. (DQ103435)

. chmie. (DQ103425)

$\operatorname{miR398a}(\mathrm{Col})$

$\operatorname{miR398b/C~(Col)~}$

\author{
CAGAGCCATTGGGAGACCTGGGACACTAGAGGCAGACA-3 \\ CCACTCGT \\ CAATAAGCCTCTTGGTGACCTGGGAACACTAGAAGCT-3' \\ AGCCACTCGGTGACCTGGGAACACTGGATGTTGACGAG-3 ' \\ AGCCTCTTGGTGACCTGGGAACACTGGATGT -3 ' \\ ACCCACTCGCTGACCTGGTTACACTCGAAGTT-3' \\ AGCCACTTGGTGACCTGGGAACACTAGAT- 3 , \\ AGCCACTTGGTGACCTGGGAACACTGGATGTTGACGAGA-3 \\ AGCCACTCGGTGACCTGGGAACACTGGATGTTG-3' \\ AGCCACTCGGTGACCTGGGAACACTGGATGT-3 \\ AGCCACTCGGTGACCTGGGAACACTG-3' \\ AGCCACTCGGTGACCTGGGAACACTGGATGTTGACGAGA-3 \\ UUCCCCACUGGACUCUUGUGU-5' \\ GUCCCCACUGGACUCUUGUGU- 5 '
}

antibody follow the same pattern (Figure 3d). From this analysis, we concluded that the levels of CCS1 transcripts and protein are linked to miR398 quantities, as already described for both CSD1 and CSD2 (Sunkar et al., 2006).

\section{An miR398-resistant form of CCS1 transcripts accumulates when miR398 is plentiful}

If CCS1 mRNAs are post-transcriptionally regulated by miR398, we hypothesized that expression of an miR398resistant form of these transcripts would result in their accumulation when copper is limiting (i.e. when miR398 levels are high). We therefore generated transgenic plants carrying an miR398-resistant form of the CCS1 cDNA driven by the CCS1 promoter and designated proCCS1::3mCCS1. We introduced three silent mutations into the miR398complementary site (Figure 4a). As a control, we also generated transgenic plants carrying the same construct with an unmodified version of the CCS1 coding sequence, designated proCCS1::CCS1. $T_{1}$ plants were selected, and, after two generations, $T_{3}$ plants containing at least one transgene in the homozygous state were grown for 12 days on medium supplemented or not with copper. CCS1 mRNA levels were determined by quantitative RT-PCR. Consistent with our previous results (Figure 3c), we observed that CCS1 transcripts are 11 times more abundant in wild-type plants grown on medium containing copper, compared with medium containing limiting amounts of copper (Figure 4b). This was also observed for four independent transgenic lines carrying the proCCS 1::CCS1 construct, but not in plants carrying proCCS1::3mCCS1 (Figure 4b). Similarly, we detected high quantities of CCS1 protein (Figure 4c) in proCCS1::3mCCS1 plants grown without copper compared to the control lines (i.e. proCCS1::CCS1 and wild-type). Both CSD1 and CSD2 proteins were undetectable under low copper conditions (Figure 4c), probably because miR398 is still mediating cleavage of the corresponding mRNAs in these plants, including proCCS $1:: 3 m C C S 1$ plants. As control, we verified that the levels of CCS1 proteins were similar in al plants grown with copper added to the medium (Figure 4c) Therefore, we concluded from this experiment and the 5'-RACE PCR mapping experiment that miR398 mediates the cleavage of CCS1 mRNA when copper is limiting.

\section{Mutations in AG010 affect the miR398-directed translational repression of CCS1 mRNAs}

Although miRNAs of plants have mainly been described as acting by triggering cleavage of their targets, Brodersen et al. (2008) demonstrated that some of these miRNAs can additionally act by translational repression, through the action of AGO1 and/or AG010 (also known as ZWILLE/ PINHEAD; ZLL). For instance, the CSD2 protein accumulates in ago10 mutants but levels of the corresponding mRNA remain almost unchanged, suggesting that $A G 010$ is probably involved in miR398-mediated translational inhibition of CSD2 mRNA (Brodersen et al., 2008). We therefore decided to quantify CCS1 mRNAs and protein in ago10 mutants. We used one of the ago 10 mutants, zll-3 (Moussian et al., 1998) in which we quantified miR398. Ten to fifteen zll-3 and wild-type plants were grown for 12 days on plates containing various amounts of copper, and total RNA was extracted in bulk. The levels of miR398 decreased when the concentrations of copper increased in the medium for both wild-type and $z / l-3$ plants (Figure 5a). By contrast, CCS 1 transcripts, quantified by quantitative RT-PCR, accumulate 
(a)

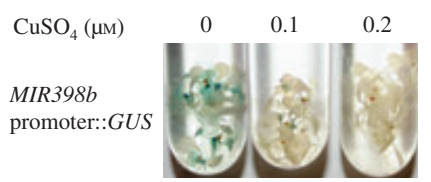

(b)

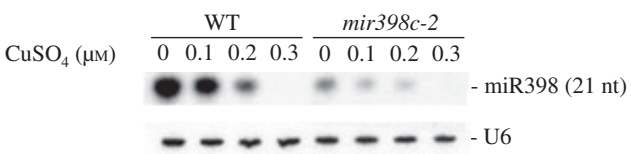

(c)

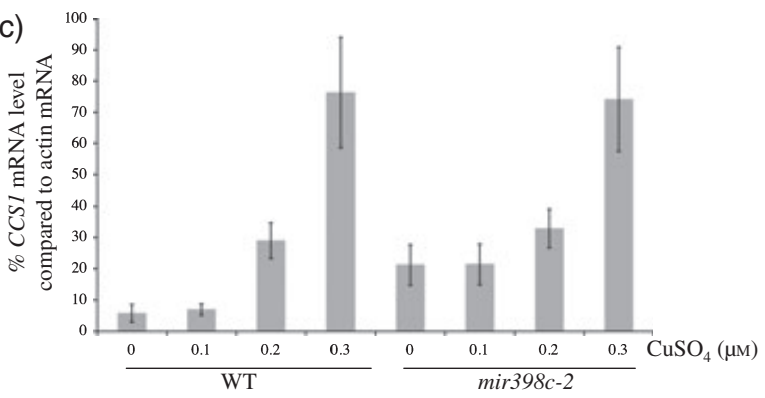

(d)

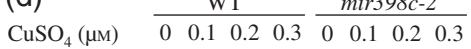

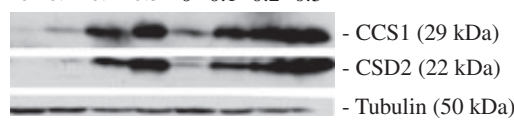

Figure 3. CCS1 is post-transcriptionally regulated by miR398.

(a) Transgenic plants expressing an MIR398b promoter::GUS transcriptional fusion. The three tubes each contain five seedlings grown on medium supplemented with various amounts of copper as indicated. GUS activity was revealed after 12 days of growth. A total of 11 independent transgenic lines were generated, showing the same pattern of expression. One representative line is shown.

(b) RNA gel blot analysis of $1 \mu \mathrm{g}$ total RNAs prepared from 12-day-old seedlings of mir398c-2 and the corresponding wild-type sibling plants grown on various amounts of copper as indicated. U6 RNA was used as a loading control.

(c) Quantitative RT-PCR of CCS1 mRNAs in the plants described in (b). Mean values for three technical PCR repeats were compared to the level of actin mRNA, arbitrarily fixed at $100 \%$. Error bars indicate SD $(n=5)$.

(d) Immunodetection of CCS1 and CSD2 proteins in the plants described in (b) using anti-CCS1 or anti-CSD2 polyclonal antibodies after SDS-PAGE of $5 \mu \mathrm{g}$ of total proteins. Detection of tubulin served as a control for equal loading.

in similar ways in wild-type and zll-3 when copper concentrations increase (Figure $5 \mathrm{~b}$ ). The same pattern was observed for CCS1 protein, as for both CSD1 and CSD2 (Figure $5 \mathrm{c}$ ). When $5 \mu \mathrm{g}$ of total proteins were separated by SDS-PAGE, CCS1, CSD1 and CSD2 protein levels were found to be slightly elevated in zll-3 mutants when copper was omitted from the medium $\left(0 \mu \mathrm{M} \mathrm{CuSO}_{4}\right)$ (Figure $5 \mathrm{c}$ ). This was further confirmed for CCS1 in both zll-1 and zll-3 alleles, when three times more proteins were loaded on the gel (Figure 5d). Thus, we concluded that the CCS1 protein, like CSD2 (Brodersen et al., 2008), is more abundant in ago10 mutants compared to wild-type plants when copper is limiting, while CCS1 mRNA and miR398 levels stay unchanged.

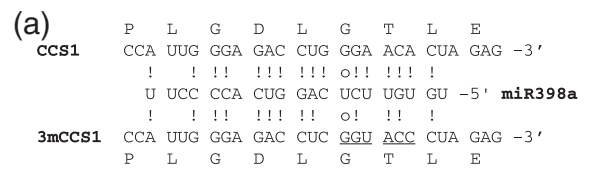

(b)

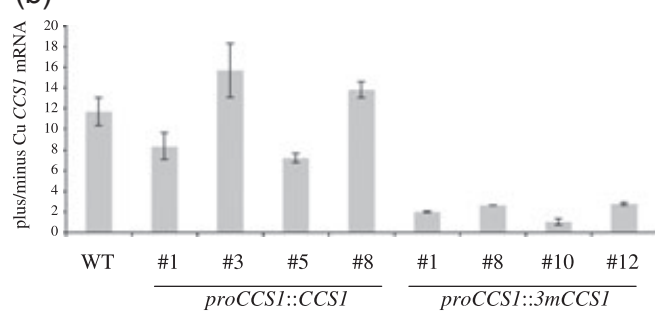

(c)

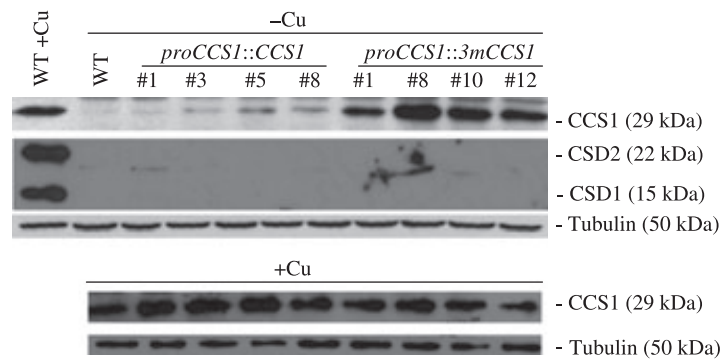

Figure 4. miR398-resistant form of CCS1 transcripts.

(a) Pairing of miR398a with the corresponding complementary sites of both CCS1 and an miR398-resistant form of CCS1 (3mCCS1). The Kpnl restriction enzyme site introduced in the mutated construct is underlined.

(b) Quantitative RT-PCR of CCS1 mRNAs in transgenic plants ( $T_{3}$ generation) carrying either unmodified CCS1 CDNA or $3 m C C S 1$ driven by the CCS1 promoter (proCCS1), and the wild-type (Col accession). Plants were grown for 12 days in vitro, with $0.5 \mu \mathrm{m}$ of $\mathrm{CuSO}_{4}$ or without copper added to the medium, and total RNAs were extracted in bulk (10-15 plants) to prepare the corresponding cDNAs and perform quantitative RT-PCR. Results are presented for four independent transgenic lines carrying proCCS 1:: CCS 1 (lines 1, 3,5 and 8 ) and four independent lines for proCCS $1:: 3$ CCS1 (lines 1, 8, 10 and 12). Values are the differences in CCS1 mRNA levels between plants grown with or without copper. Error bars indicate $\operatorname{SD}(n=3)$.

(c) Immunodetection of CCS1, CSD1 and CSD2 proteins in $15 \mu \mathrm{g}$ of total proteins extracted from the plants described in (b). Proteins were detected using anti-CCS1 or anti-CSD1/2 polyclonal antibodies after SDS-PAGE. Detection of tubulin served as a control for equal loading.

\section{DISCUSSION}

In the present study, we analysed the transcriptomes of two mir398 T-DNA mutants containing lower and higher contents of miR398 to find targets that had not previously been identified by bioinformatics predictions. In addition to CSD1 and CSD2 mRNAs, we identified CCS1 mRNAs encoding the chaperone that delivers copper to the $\mathrm{Cu} / \mathrm{Zn}$ SODs of Arabidopsis. To demonstrate that miR398 triggers the cleavage of CCS 1 mRNAs, we mapped the $5^{\prime}$ end of the cleavage products in the miR398-complementary site of CCS1. Then we generated transgenic plants carrying a CCS1 cDNA form that was resistant to cleavage by miR398, and found that CCS1 protein accumulates in these plants when the miRNA is abundant. Thus, we concluded from these experiments 

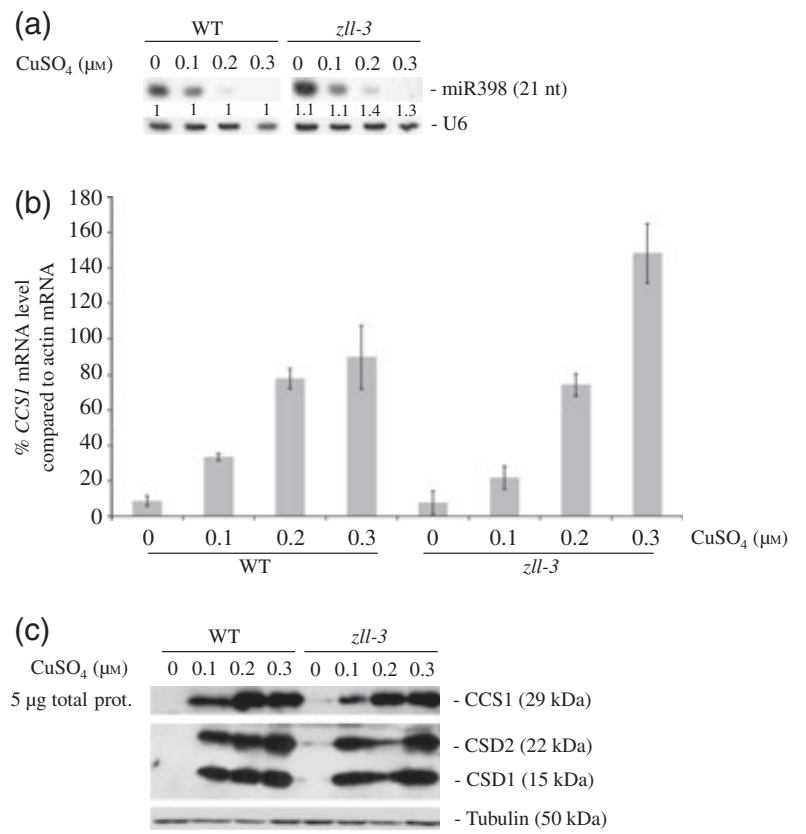

(d) $0 \mu_{\mathrm{m}} \mathrm{CuSO}_{4}$

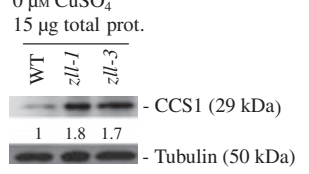

Figure 5. ago 10 mutants are impaired in miR398-directed post-transcriptional regulation of CCS1.

(a) RNA gel blot analysis of $1 \mu \mathrm{g}$ total RNAs prepared from 12-day-old seedlings of zll-3 mutants and the corresponding wild-type (Ler). Plants were grown on various amounts of copper as indicated. U6 RNA was used as a loading control, and values for miR398 normalized to those for U6 RNA are indicated. Values for wild-type controls were set at 1.

(b) Quantitative RT-PCR of CCS1 mRNAs in the plants described in (a). Mean values for three technical PCR repeats were compared to the level of actin mRNA, arbitrarily fixed at $100 \%$. Error bars indicate $\mathrm{SD}(n=5)$.

(c) Immunodetection of CCS1, CSD1 and CSD2 proteins in the plants described in (a) using anti-CCS1 or anti-CSD2/CSD1 polyclonal antibodies after SDS-PAGE of $5 \mu \mathrm{g}$ of total proteins. Detection of tubulin served as a control for equal loading.

(d) Immunodetection of CCS1 in $15 \mu \mathrm{g}$ of total proteins extracted from 12-dayold seedlings of $z$ Il-3 or zll-1 mutants and the corresponding wild-type (Ler). Plants were grown without copper. CCS1 was detected using an anti-CCS1 polyclonal antibody after SDS-PAGE. Detection of tubulin served as a control for equal loading. Normalized values of CCS1 protein to tubulin controls (with wild-type controls set at 1.0 ) are indicated.

that miR398 mediates the cleavage of CCS1 mRNA when copper is limiting. In consequence, miR398 is controlling the level of both CSD1 and CSD2 post-transcriptionally by two means: directly by directing the slicing of their mRNAs, and indirectly by triggering cleavage of the mRNA encoding the copper chaperone necessary for their activation (Figure 6). We found no developmental phenotypes associated with the production of a CCS1 miR398-resistant form when CCS1 is normally down-regulated in copper limiting conditions. Similarly, null ccs 1 mutants, in which $\mathrm{Cu} / \mathrm{Zn}$ SOD activities

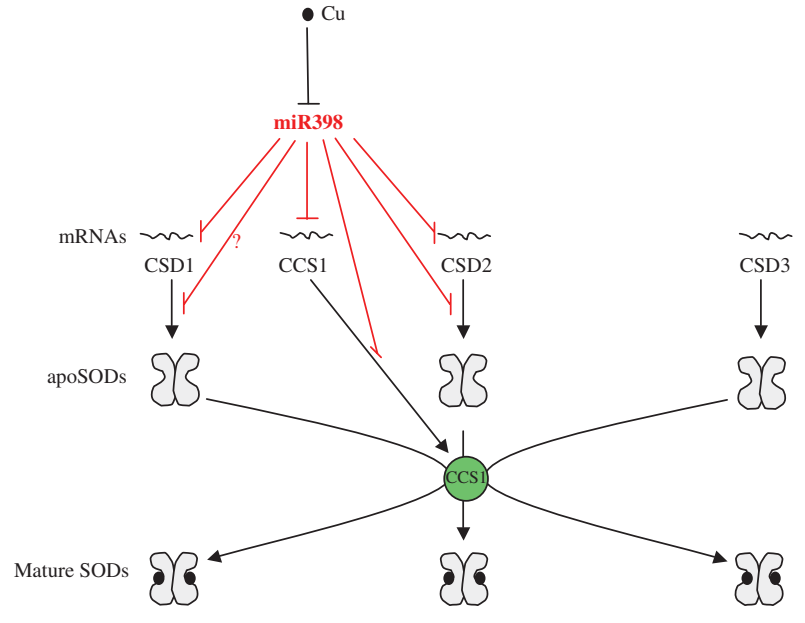

Figure 6. miR398 controls the production of $\mathrm{Cu} / \mathrm{Zn}$ SODs.

CSD1, CSD2 and CSD3 are the three $\mathrm{Cu} / \mathrm{Zn}$ SODs of Arabidopsis, and are localized in the cytoplasm, chloroplast and peroxisome, respectively. miR398 controls the production of both CSD1 and CSD2 mRNAs by mediating their cleavage and production of the corresponding apoproteins by translational repression, at least for CSD2. miR398 also controls the production of CCS1, the chaperone delivering copper to all $\mathrm{Cu} / \mathrm{Zn}$ SODs, at both the mRNA and protein levels.

are almost undetectable (Chu et al., 2005), present no particular phenotypes (N.B., data not shown). Therefore, it is probable that miR398 action on CCS1, CSD1 and CSD2, when copper is absent, is crucial in stress situations that remained to be identified.

In previous studies, plants expressing miR398-resistant forms of CSD1 or CSD2 (five mutations introduced into the miR398-complementary sites) were shown to accumulate the corresponding mRNAs when miR398 is abundant, but, surprisingly, both CSD1 and CSD2 protein levels remained unchanged (Dugas and Bartel, 2008). The authors concluded that, despite the absence of cleavage, miR398 was able to repress translation of the mutated CSD1 or CSD2 mRNAs. All three $\mathrm{Cu} / \mathrm{Zn}$ SOD isoforms of Arabidopsis, including both CSD1 and CSD2, are nearly absent in a null ccs 1 knockout mutant (Chu et al., 2005), with almost no detectable Cu/Zn SOD activities, and very low amounts of proteins detected by Western blotting (Chu et al., 2005). This indicates that CCS1 is necessary for the activation of both CSD1 and CSD2 apo-forms, as found previously for SODs of yeast cells (Culotta et al., 1997) and Drosophila (Kirby et al., 2008). In plants expressing mutated CSD mRNA forms, miR398 still triggers the cleavage of CCS1 mRNAs, probably explaining why CSD1 or CSD2 mRNAs accumulate, but not the corresponding proteins. In contrast, in transgenic plants expressing a CCS1 mRNA version resistant to cleavage by miR398, we observed that both CCS1 mRNA and protein accumulate (Figure 4).

The regulation of miRNA targets differs in several ways between animals and plants (Brodersen and Voinnet, 2009). 
In animals, miRNAs mainly cause translational repression of target mRNAs by pairing their 3' UTRs, often at multiple sites. The target site of most animal miRNAs is short (only 6-8 bases), with perfect pairing between the miRNA and its target. By contrast, plant miRNAs have a long target site, usually in mRNA coding regions, with extensive near-perfect pairing, and act mainly by triggering cleavage of the target mRNA. Thus, the requirements for sequence complementarity between miRNAs and mRNA target sites differ between plants and animals, explaining why the number of predicted plant miRNA targets is relatively low (JonesRhoades and Bartel, 2004). Likewise, the number of targets revealed by our transcriptome analyses (designed to identify cleaved targets) is also very limited. Indeed, we found only one mRNA target, namely CCS1, that was not previously identified by bioinformatics approaches, in addition to CSD1 and CSD2 mRNAs. In agreement with previous observations (Dugas and Bartel, 2008), we found no differences in the expression level of $C O X 5 b-1$, a predicted target of miR398 confirmed by 5'-RACE PCR (Jones-Rhoades and Bartel, 2004). Nevertheless, we cannot exclude the possibility that COX5b-1, or other mRNAs, are stress-specific miR398 targets that were not revealed by our transcriptome analyses performed with plants grown under standard conditions. Alternatively, some targets might only be regulated by miR398-directed translational repression.

CCS1 protein accumulates in ago10 mutants (Figure 5), suggesting that $A G 010$ is involved in miR398-directed posttranscriptional regulation of CCS1. miR398 acts through translational repression and cleavage of at least two of its mRNA targets identified so far: CSD2 and CCS1 (Figure 6). Now that targets of plant miRNAs regulated by translational repression are being discovered, the picture becomes more complicated, as at least some miRNAs of plants are involved in triggering both cleavage and translational repression. Plant mRNA targets specifically regulated by translational repression remain to be identified by future studies, for instance by analysing the proteomes of mutants impaired in the production of miRNAs.

Finally, a crucial point about CCS1 is to understand why CCS1 mRNA escaped all bioinformatics predictions, and why the miR398 target site was not discovered previously. To identify real targets and minimize the number of false positives, the first prediction algorithms used were very restrictive, allowing only three mismatches between the miRNA and the putative mRNA target, with no bulges in the duplex, and treating GU wobbles as mismatches (Rhoades et al., 2002). Using a system of penalty scoring that takes into account GU wobbles and bulges, and restricting the results to target sequences conserved between species, more targets could be predicted and validated (Jones-Rhoades and Bartel, 2004). Finally, additional targets have been found (Wang et al., 2004; Allen et al., 2005; Schwab et al., 2005; Rajagopalan et al., 2006; Fahlgren et al., 2007; Alves-Junior et al., 2009) using more sophisticated algorithms that take into consideration further parameters such as the minimum free energy of the duplex, expression profiles of the putative targets in miRNA biogenesis mutants, and the position of the mismatches, as the pairing in the $5^{\prime}$ seed region of the miRNA and around the cleavage site (spanning nucleotides 10 and 11) appears to be the most important (Schwab et al., 2005; Mallory and Bouché, 2008). About 120 Arabidopsis miRNA/target mRNA duplexes have been validated by 5 -RACE PCR since the discovery of miRNAs in plants (Table S1), and we found only six duplexes that exceed four mismatches in this list (if we consider GU as a mismatch). Five of these, the duplexes formed by miR-JAW and TCP3, 4 or 10 (Palatnik et al., 2007), the one formed by miR159a and CSD3 (German et al., 2008), and finally the one between miR398 and CCS1 mRNA, correspond to target genes identified experimentally. The targets of miR-JAW and miR398 were found by analysing the transcriptomes of mir-jaw (Palatnik et al., 2007) and mir398 mutants (Table 1), respectively, and CSD3 was identified as a target of miR159 by analysing the degradome of Arabidopsis (German et al., 2008). The only exception is from a recent study (AlvesJunior et al., 2009), in which a target of miR161 containing six GU wobbles and two mismatches was predicted and validated by 5'-RACE PCR. In this study, GU wobbles were not considered as mismatches, but the predicted energy of the duplex was used as a filter. Such new algorithms, allowing more than four mismatches, and applying more flexible rules to the pairing in the seed region and the GU content, combined with transcriptomic analyses of mutants impaired in the production of miRNAs, are likely to result in discovery of new targets.

\section{EXPERIMENTAL PROCEDURES}

\section{Plant materials and growth conditions}

Arabidopsis thaliana ecotypes Columbia (Col), Landsberg erecta (Ler) and Wassilewskija (Ws) were used. Surface-sterilized seeds were plated on culture medium (Appendix S1) (Estelle and Somerville, 1987) containing $0.8 \%$ agar (plant cell culture tested, Sigma, http://www.sigmaaldrich.com/), incubated at $4^{\circ} \mathrm{C}$ for $48 \mathrm{~h}$, and grown in vitro under the following conditions: day/night cycles of $16 / 8 \mathrm{~h}$; light intensity $100-150 \mu \mathrm{mol} \mathrm{m}^{-2} \mathrm{sec}^{-1}$, temperature day/ night $20 / 15^{\circ} \mathrm{C}$; humidity $65 \%$.

The Ws mir398c-2 mutant (FLAG_072C02) was isolated from the INRA (Institut National de la Recherche Agronomique, Versailles, France) collection of T-DNA mutants and genotyped using forward (5'-CCTAGTACCTCTTTGACGTTC- ${ }^{\prime}$ ) and reverse ( $5^{\prime}$-GCGTTGA TAAAACATGGACGG-3') primers specific to the MIR398c gene, and the T-DNA primer TAG5 (5'-CTACAAATTGCCTTTTCTTATCGAC-3'). The Col-0 mir398c-3 mutant (SALK_017104) was isolated from the Salk Institute collection of T-DNA mutants, and genotyped using forward (5'-CCTAGTACCTCTTTGACGTTC- $3^{\prime}$ ) and reverse (5'-CACAATCGTGTTGATGAGCTC-3') primers specific to the MIR398C gene, and T-DNA primer LBa1 (5'-ATGGTTCACGTAGTGGGCCATC-3'). zll (ago10) EMS mutants have been described previously (Moussian et al., 1998). 


\section{Generation of transgenic plants}

To generate plants carrying an miR398-resistant form of CCS1, we cloned the CCS1 promoter in front of the CCS1 cDNA in pBIB-Hygro (Becker, 1990). The CCS1 cDNA was amplified from clone pda15533 (Riken Institute; GenBank accession number AK221656) using forward primer 5'-ATTCTAGACTCGAATTCGTCGAGTTAAT-3' (Xbal site underlined) and reverse primer 5'-GACTGCGGCCGCCAAAACAAGTTCTCAATCAC-3' (Notl site underlined), and transferred to pBluescript KS+ (Stratagene, http://www.stratagene.com) at the corresponding sites. Clones were sequenced and then mutagenized using a QuikChange site-directed mutagenesis kit (Stratagene) and primers 5 '-CCATTGGGAGACCTCGGTACCCTAGAGGCAGAC-3' (forward) and 5'-GTCTGCCTCTAGGGTACCGAGGTCTCCCAATGG-3' (reverse), which introduce a Kpnl site (underlined). In parallel, the promoter of CCS1 (approximately $2 \mathrm{~kb}$ upstream of the first ATG) was amplified from Col genomic DNA and cloned into a pBIB-Hygro binary vector using primers 5 '-GGAAGTTGTAGAGAAGCTTAAGG- 3 ' (forward, native Hindlll site underlined) and $5^{\prime}$-GTCATCTAGACTTGCTTCTTCAGTTTTTGCGG-3' (reverse, Xbal site underlined). CCS1 and $3 m C C S 1 \mathrm{cDNAs}$ were then transferred from pBluescript to $\mathrm{pBIB}$ Hygro by digestion with $X b a l$ and Sacl to obtain the proCCS1::CCS1 and proCCS1::3mCCS1 constructs.

For miR398b promoter::GUS constructs, we amplified $2 \mathrm{~kb}$ upstream of the predicted fold-back structure from Col genomic DNA using forward primer 5'-ACGTAAGCTTTCTAAACCTAAAGAAACCTTAAG-3' (Hindlll site underlined) and reverse primer 5'-ACGTCTAGACAACCCTGTCGAGATCCACTACC-3' (Xbal site underlined). The PCR product was cloned into the corresponding sites of pBIB-Hygro, and the clones were sequenced.

\section{RNA analysis}

Total RNA was extracted as described previouslye (Bouché et al., 2006). Small RNAs were separated by electrophoresis on $15 \%$ polyacrylamide gels and blotted onto membranes. The miR398 ${ }^{32}$ P-end-labelled oligonucleotide probe was 5'-CAGGGGTGACCTGAGAACACA-3'. RNA hybridization signals were quantified using a Fuji phosphorimager (Fuji, http://www.fujifilm.com).

For the analyses of mRNAs expression by quantitative RT-PCR, total RNA extraction was performed using an RNeasy plant kit (Qiagen, http://www.qiagen.com/), followed by a DNase treatment (Fermentas, http://www.fermentas.com). RT-PCR was performed on $500 \mathrm{ng}$ of total RNAs using M-MLV reverse transcriptase (Fermentas), and cDNAs were diluted 10 times. A $5 \mu$ laliquot was used for quantitative RT-PCR using a MasterCycler ep realplex (Eppendorf) with RealMasterMix SYBR ROX/SYBR solutions (5 PRIME, http:// www.5prime.com) using the primers $5^{\prime}$-CCCATATGACAGTACCATCA-3' (forward) and 5'- CCATTTCAAGATCAAACTGGCAC- $3^{\prime}$ (reverse) for CCS1, and 5'-GCACCCTGTTCTTCTTACCG-3' (forward) and $5^{\prime}$-AACCCTCGTAGATTGGCACA-3' (reverse) for actin.

To identify the miR398 cleavage site in CCS1 mRNA, we used a GeneRacer kit (Invitrogen, http://www.invitrogen.com/) as previously described (Llave et al., 2002).

\section{Western blotting}

Polyclonal antibodies against recombinant CSD2 or CCS1 proteins were obtained from Agrisera (http://www.sera.com) and used according to the manufacturer's instructions. Total proteins

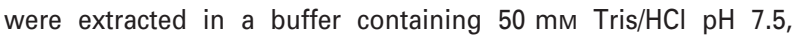
$150 \mathrm{~mm} \mathrm{NaCl}, 10 \%$ glycerol, separated by SDS-PAGE, and transferred to PVDF membranes. Blocking was performed in Tris Buffered Saline Tween-20 (T-BST) containing 5\% milk. Antibodies against CSD2 were also used to detect CSD1 (dilution of 1/1000)
(Kliebenstein et al., 1998, 1999). Tubulin was detected using monoclonal anti- $\alpha$-tubulin (Sigma; reference number T5168) according to the manufacturer's instructions. Proteins were revealed using a Western Lightning kit (PerkinElmer Life Sciences, http://www.perkinelmer.com).

\section{Transcriptome studies and statistical analysis of microarray data}

Microarray analysis was performed using the CATMA array (version 2.2) containing 24576 gene-specific tags corresponding to 22089 genes from Arabidopsis (Crowe et al., 2003; Hilson et al., 2004). For each genotype, two independent biological replicates were used. Total RNA extraction was performed using an RNeasy plant kit (Qiagen) as indicated by the manufacturer. RNA integrity, cDNA synthesis, hybridization and array scanning were performed as described previously (Lurin et al., 2004).

Statistical analysis was based on two dye swaps (i.e. four arrays, each containing 24576 gene-specific tags and 384 controls) as described previously (Gagnot et al., 2008). To identify the genes that were differentially expressed, we performed a paired $t$ test on the log ratios, assuming that the variance of the log ratios was the same for all genes. Spots displaying extreme variance (too small or too large) were excluded. The raw $P$ values were adjusted by the Bonferroni method, which controls the family-wise error rate. We considered genes with a Bonferroni $P$ value lower than $5 \%$ as being differentially expressed, as described previously (Gagnot et al., 2008). We used the Bonferroni method (with a type I error equal to $5 \%$ ) in order to maintain strong control of the false positives in a multiple-comparison context (Ge et al., 2003). The microarray data are available at the following address; http://urgv.evry.inra.fr/CATdb.

\section{ACKNOWLEDGEMENTS}

Research costs were met by the Institut National de la Recherche Agronomique. The authors wish to thank the Salk Institute Genomic Analysis Laboratory for providing the sequence-indexed Arabidopsis T-DNA insertion mutants and Thomas Laux for providing the zll mutants. We thank Isabelle Gy and Vincent Jauvion for helpful discussions and for critical reading of the manuscript. We thank Hervé Vaucheret and his group for their support and fruitful discussions, especially Allison Mallory.

\section{SUPPORTING INFORMATION}

Additional Supporting Information may be found in the online version of this article:

Figure S1. Expression of MIR398 precursors in miR398 mutants.

Figure S2. Nucleotide pairing between miR398 and target mRNAs presented in Table 1.

Figure S3. CCS1 mRNAs are post-transcriptionally regulated by miR398 when sucrose is added to the medium.

Table S1. miRNA/mRNAs duplexes validated by $5^{\prime}$-RACE-PCR.

Appendix S1. Supplemental details for the in vitro culture medium. Please note: As a service to our authors and readers, this journal provides supporting information supplied by the authors. Such materials are peer-reviewed and may be re-organized for online delivery, but are not copy-edited or typeset. Technical support issues arising from supporting information (other than missing files) should be addressed to the authors.

\section{REFERENCES}

Abdel-Ghany, S.E., Burkhead, J.L., Gogolin, K.A., Andres-Colas, N., Bodecker, J.R., Puig, S., Penarrubia, L. and Pilon, M. (2005) AtCCS is a functional 
homolog of the yeast copper chaperone Ccs1/Lys7. FEBS Lett. 579, 23072312.

Allen, E., Xie, Z., Gustafson, A.M. and Carrington, J.C. (2005) microRNAdirected phasing during trans-acting siRNA biogenesis in plants. Cell, 121, 207-221.

Alves-Junior, L., Niemeier, S., Hauenschild, A., Rehmsmeier, M. and Merkle, T. (2009) Comprehensive prediction of novel microRNA targets in Arabidopsis thaliana. Nucleic Acids Res. 37, 4010-4021.

Bonnet, E., Wuyts, J., Rouze, P. and Van de Peer, Y. (2004) Detection of 91 potential conserved plant microRNAs in Arabidopsis thaliana and Oryza sativa identifies important target genes. Proc. Natl Acad. Sci. USA, 101 11511-11516.

Bouché, N., Lauressergues, D., Gasciolli, V. and Vaucheret, H. (2006) An antagonistic function for Arabidopsis DCL2 in development and a new function for DCL4 in generating viral siRNAs. EMBO J. 25, 3347-3356.

Brodersen, P. and Voinnet, O. (2009) Revisiting the principles of microRNA target recognition and mode of action. Nature Rev. Mol. Cell Biol. 10, 141148.

Brodersen, P., Sakvarelidze-Achard, L., Bruun-Rasmussen, M., Dunoyer, P., Yamamoto, Y.Y., Sieburth, L. and Voinnet, O. (2008) Widespread translational inhibition by plant miRNAs and siRNAs. Science, 320, 1185-1190.

Chiou, T.J. (2007) The role of microRNAs in sensing nutrient stress. Plant Cell Environ. 30, 323-332.

Chu, C.C., Lee, W.C., Guo, W.Y., Pan, S.M., Chen, L.J., Li, H.M. and Jinn, T.L. (2005) A copper chaperone for superoxide dismutase that confers three types of copper/zinc superoxide dismutase activity in Arabidopsis. Plant Physiol. 139, 425-436.

Crowe, M.L., Serizet, C., Thareau, V. et al. (2003) CATMA: a complete Arabidopsis GST database. Nucleic Acids Res. 31, 156-158.

Culotta, V.C., Klomp, L.W., Strain, J., Casareno, R.L., Krems, B. and Gitlin, J.D. (1997) The copper chaperone for superoxide dismutase. J. Biol. Chem. 272, 23469-23472.

Dugas, D.V. and Bartel, B. (2008) Sucrose induction of Arabidopsis miR398 represses two $\mathrm{Cu} / \mathrm{Zn}$ superoxide dismutases. Plant Mol. Biol. 67, 403-417.

Estelle, M.A. and Somerville, C. (1987) Auxin-resistant mutants of Arabidopsis thaliana with an altered morphology. Mol. Gen. Genet. 206, 200-206.

Fahlgren, N., Howell, M.D., Kasschau, K.D. et al. (2007) High-throughput sequencing of Arabidopsis microRNAs: evidence for frequent birth and death of miRNA genes. PLOS ONE, 2, e219.

Gagnot, S., Tamby, J.P., Martin-Magniette, M.L., Bitton, F., Taconnat, L. Balzergue, S., Aubourg, S., Renou, J.P., Lecharny, A. and Brunaud, V. (2008) CATdb: a public access to Arabidopsis transcriptome data from the URGV-CATMA platform. Nucleic Acids Res. 36, D986-D990.

Ge, S., Dudoit, S. and Speed, T.P. (2003) Resampling-based multiple testing for microarray data analysis. TEST, 12, 1-44.

German, M.A., Pillay, M., Jeong, D.H. et al. (2008) Global identification of microRNA-target RNA pairs by parallel analysis of RNA ends. Nat. Biotechnol. 26, 941-946.

Hilson, P., Allemeersch, J., Altmann, T. et al. (2004) Versatile gene-specific sequence tags for Arabidopsis functional genomics: transcript profiling and reverse genetics applications. Genome Res. 14, 2176-2189.

Jagadeeswaran, G., Saini, A. and Sunkar, R. (2009) Biotic and abiotic stress down-regulate miR398 expression in Arabidopsis. Planta, 229, 1009-1014.

Jia, X., Wang, W.X., Ren, L., Chen, Q.J., Mendu, V., Willcut, B., Dinkins, R., Tang, X. and Tang, G. (2009) Differential and dynamic regulation of miR398 in response to ABA and salt stress in Populus tremula and Arabidopsis thaliana. Plant Mol. Biol. 71, 51-59.

Jones-Rhoades, M.W. and Bartel, D.P. (2004) Computational identification of plant microRNAs and their targets, including a stress-induced miRNA. Mol. Cell, 14, 787-799.
Kirby, K., Jensen, L.T., Binnington, J., Hilliker, A.J., Ulloa, J., Culotta, V.C. and Phillips, J.P. (2008) Instability of superoxide dismutase 1 of Drosophila in mutants deficient for its cognate copper chaperone. J. Biol. Chem. 283 35393-35401.

Kliebenstein, D.J., Monde, R.A. and Last, R.L. (1998) Superoxide dismutase in Arabidopsis: an eclectic enzyme family with disparate regulation and protein localization. Plant Physiol. 118, 637-650.

Kliebenstein, D.J., Dietrich, R.A., Martin, A.C., Last, R.L. and Dangl, J.L. (1999) LSD1 regulates salicylic acid induction of copper zinc superoxide dismutase in Arabidopsis thaliana. Mol. Plant Microbe Interact. 12, 1022-1026.

Kruger, J. and Rehmsmeier, M. (2006) RNAhybrid: microRNA target prediction easy, fast and flexible. Nucleic Acids Res. 34, W451-W454.

Lanet, E., Delannoy, E., Sormani, R., Floris, M., Brodersen, P., Crete, P., Voinnet, O. and Robaglia, C. (2009) Biochemical evidence for translationa repression by Arabidopsis microRNAs. Plant Cell, 21, 1762-1768.

Llave, C., Xie, Z., Kasschau, K.D. and Carrington, J.C. (2002) Cleavage of Scarecrow-like mRNA targets directed by a class of Arabidopsis miRNA. Science, 297, 2053-2056.

Lurin, C., Andres, C., Aubourg, S. et al. (2004) Genome-wide analysis of Arabidopsis pentatricopeptide repeat proteins reveals their essential role in organelle biogenesis. Plant Cell, 16, 2089-2103.

Mallory, A.C. and Bouché, N. (2008) MicroRNA-directed regulation: to cleave or not to cleave. Trends Plant Sci. 13, 359-367.

Mallory, A.C., Hinze, A., Tucker, M.R., Bouché, N., Gasciolli, V., Elmayan, T., Lauressergues, D., Jauvion, V., Vaucheret, H. and Laux, T. (2009) Redundant and specific roles of the ARGONAUTE proteins AGO1 and ZLL in development and small RNA-directed gene silencing. PLoS Genet. 5, e1000646.

Moussian, B., Schoof, H., Haecker, A., Jurgens, G. and Laux, T. (1998) Role of the ZWILLE gene in the regulation of central shoot meristem cell fate during Arabidopsis embryogenesis. EMBO J. 17, 1799-1809.

Palatnik, J.F., Wollmann, H., Schommer, C. et al. (2007) Sequence and expression differences underlie functional specialization of arabidopsis microRNAs miR159 and miR319. Dev. Cell, 13, 115-125.

Rajagopalan, R., Vaucheret, H., Trejo, J. and Bartel, D.P. (2006) A diverse and evolutionarily fluid set of microRNAs in Arabidopsis thaliana. Genes Dev. 20, 3407-3425.

Rehmsmeier, M., Steffen, P., Hochsmann, M. and Giegerich, R. (2004) Fast and effective prediction of microRNA/target duplexes. RNA, 10, 1507-1517.

Rhoades, M.W., Reinhart, B.J., Lim, L.P., Burge, C.B., Bartel, B. and Bartel, D.P (2002) Prediction of plant microRNA targets. Cell, 110, 513-520.

Schwab, R., Palatnik, J.F., Riester, M., Schommer, C., Schmid, M. and Weigel D. (2005) Specific effects of microRNAs on the plant transcriptome. Dev. Cell, 8, 517-527.

Sunkar, R. and Zhu, J.K. (2004) Novel and stress-regulated microRNAs and other small RNAs from Arabidopsis. Plant Cell, 16, 2001-2019.

Sunkar, R., Kapoor, A. and Zhu, J.K. (2006) Posttranscriptional induction of two $\mathrm{Cu} / \mathrm{Zn}$ superoxide dismutase genes in Arabidopsis is mediated by downregulation of miR398 and important for oxidative stress tolerance. Plant Cell, 18, 2051-2065.

Trindade, I., Capitao, C., Dalmay, T., Fevereiro, M.P. and Santos, D.M. (2010) miR398 and miR408 are up-regulated in response to water deficit in Medicago truncatula. Planta, 231, 705-716.

Voinnet, 0. (2009) Origin, biogenesis, and activity of plant microRNAs. Cell 136, 669-687.

Wang, X.J., Reyes, J.L., Chua, N.H. and Gaasterland, T. (2004) Prediction and identification of Arabidopsis thaliana microRNAs and their mRNA targets. Genome Biol. 5, R65.

Yamasaki, H., Abdel-Ghany, S.E., Cohu, C.M., Kobayashi, Y., Shikanai, T. and Pilon, M. (2007) Regulation of copper homeostasis by micro-RNA in Arabidopsis. J. Biol. Chem. 282, 16369-16378. 